\title{
Is Testing for Rapid Virological Response (RVR) Necessary in the Present Era of Treatment of Hepatitis C? : An Analysis from South Asia
}

\author{
Ruchir Patel $^{1}$, Alisha Chaubal ${ }^{1}$, Pathik Parikh ${ }^{2}$, Chetan Rathi ${ }^{1}$, Meghraj Ingle ${ }^{3}$, Prabha Sawant ${ }^{4}$ \\ ${ }^{1}$ Resident, Department of Gastroenterology, Lokmanya Tilak Municipal Medical College \& Hospital, Mumbai \\ ${ }^{2}$ Lecturer, Department of Gastroenterology, Lokmanya Tilak Municipal Medical College \& Hospital, Mumbai \\ ${ }^{3}$ Associate professor, Department of Gastroenterology, Lokmanya Tilak Municipal Medical College \& Hospital, Mumbai \\ ${ }^{4}$ Professor and Head, Department of Gastroenterology, Lokmanya Tilak Municipal Medical College \& Hospital, Mumbai, India
}

\begin{abstract}
Received: August 12, 2016; Accepted: August 28, 2016; Published: September 13, 2016
*Corresponding author: Ruchir Patel, Department of Gastroenterology, Lokmanya Tilak Municipal Medical College \& Hospital, Sion, Mumbai, 400022, Tel no: +917506065561; 022-24063088; Email: ruchir.cn@gmail.com
\end{abstract}

\begin{abstract}
Background and aims: Treatment of hepatitis $\mathrm{C}$ has become revolutionised with the availability of newer direct acting antiviral (DAA) in last two years. In India, Sofosbuvir was the only available DAA till December 2015. However affordability remains the major hurdle in the hepatitis C treatment in India. So we assessed whether testing for HCV RNA (hepatitis C virus ribonucleic acid) at 4 weeks can be skipped or not and make the treatment more cost-effective especially in India.
\end{abstract}

Methods: We retrospectively evaluated RVR, ETR (end of treatment response) and SVR12 (sustained virological response) rates in patients with chronic hepatitis $\mathrm{C}$ treated with sofosbuvir containing regimens in our centre and assessed the relation between RVR and SVR12. We also reviewed the available trials of similar regimens to prove our hypothesis.

Results: In our cohort, 100\% (30/30) patients achieved RVR, ETR and SVR12 irrespective of genotype, presence of cirrhosis or previous treatment history. Other trials also showed very high RVR rates (94$100 \%$ ) with SVR12 rate $>90 \%$. Substandard treatment given in few trials like Sofosbuvir monotherapy in genotype 3 and Sofosbuvir + Ribavirin in treatment experienced genotype 1 had SVR12 rates of $60 \%$ and $10 \%$ respectively despite of $100 \%$ RVR rate.

Conclusions: Till date, no data has emerged to suggest that those with detectable HCV RNA at 4 weeks will not attain SVR. Pretreatment HCV RNA and demonstrating its absence at 12 weeks after the end of therapy may suffice in treatment with Sofosbuvir especially in the resource poor countries like India.

Keywords: Rapid virological response; Sofosbuvir; Response guided treatment; Hepatitis C; Direct acting antivirals

\section{Background}

Treatment of hepatitis $\mathrm{C}$ has evolved in the last few years with the availability of newer direct acting antivirals (DAA) like NS3/4A protease inhibitors, NS5A inhibitors and NS5B polymerase inhibitors. Trials of these newer DAAs have shown tremendous success rates [1]. At present, recommendations on monitoring of hepatitis $C$ treatment are sparse. Most of the guidelines recommend to monitor HCV RNA (hepatitis C virus ribonucleic acid) at week 4 and at the end of treatment as well as 12 or 24 weeks post treatment in treatment with DAAs [1, 2]. Rapid virological response (RVR) is defined as undetectable $(<50 \mathrm{IU} / \mathrm{ml}) \mathrm{HCV}$ RNA at week 4 of starting treatment. It was the strongest predictor of Sustained Virological Response (SVR) in patients treated with Pegylated Interferon (PEG-IFN) and Ribavirin [3]. Along with few pre-treatment parameters, RVR was used for shortening or prolonging the therapy with PEG-IFN and Ribavirin [4].However, there are no studies till date showing RVR as a predictor of SVR in treatment with DAAs.

In India, only available DAA till the end of 2015 was Sofosbuvir. Hepatitis C is more prevalent in lower socioeconomic class [5] and affordability is a major obstacle in treatment of hepatitis $\mathrm{C}$ especially in the resource poor countries like India. Skipping the HCV RNA test at 4 weeks could be one of the possible ways to make the therapy more cost effective. Here we studied the response rates of treatment with Sofosbuvir containing regimens from our hospital and compared with other available studies.

\section{Aims and Objectives}

We hypothesized that testing for RVR is not necessary in the era of Sofosbuvir. To prove the hypothesis, we put the following objectives in our study [1]. To study the response rates [RVR, end of treatment response (ETR) and SVR] of hepatitis C treatment containing either Sofosbuvir, PEG-IFN and Ribavirin (SPR regimen) or Sofosbuvir and Ribavirin (SR regimen) [2]. To study the patterns of RVR, ETR and SVR in relation to various genotypes, presence of cirrhosis and history of previous treatment [3]. To compare with the other available trials of these regimens.

\section{Methods}

We retrospectively evaluated the patients with chronic hepatitis $\mathrm{C}$ who were given either of the two Sofosbuvir containing 
regimens in the department of gastroenterology in a tertiary care municipal hospital in Mumbai from May 2015 (availability of Sofosbuvir in the Indian market) to November 2015. All patients with chronic hepatitis $\mathrm{C}$ with age $>18$ years, with any detectable viral load, any genotype, with or without cirrhosis, treatment naïve or experienced were included in the analysis. Except for the decompensated cirrhosis, all patients were given triple therapy containing Sofosbuvir $400 \mathrm{mg}$ once a day, PEG-IFN $\alpha 2 \mathrm{a} 180 \mu \mathrm{g}$ subcutaneously once a week and Ribavirin daily in two divided doses (weight based, $1000 \mathrm{mg}$ if $<75 \mathrm{~kg}, 1200 \mathrm{mg}$ if $>75 \mathrm{~kg}$ ) for 12 weeks. All decompensated cirrhotic patients were given Sofosbuvir (400 mg daily) and low dose Ribavirin $600 \mathrm{mg}$ daily for 24 weeks. Patients with HIV or hepatitis B co-infection and those who have not completed the therapy were excluded from the analysis. Therapy was monitored with HCV RNA levels at 4 weeks, 12 weeks during treatment and 12 weeks post treatment; complete hemogram every week, renal and liver function tests every month, thyroid function tests pre and post treatment.RVR, ETR and SVR12 were calculated according to genotype, cirrhosis status and history of previous treatment. RVR and SVR12 rates of other available trials were also analysed.

\section{Results}

Total 45 patients were given SPR regimen. Out of which 30 patients completed the therapy till date; hence included in the analysis. Two patients lost to follow up. Rest are yet to complete the therapy. Four patients were given SR treatment, but 3 of them lost to follow-up; 2 lost follow-up before 24 weeks of therapy and 1 in the first week post therapy. One patient stopped treatment in the $8^{\text {th }}$ week. None of them were included in the analysis. Baseline characteristics of the patients are shown in the table 1. Majority of the patients were female (80\%, n-24). 17 (57\%) patients were having cirrhosis. Seventeen (57\%), 12 (40\%) and $1(3 \%)$ patients were having genotype 3,1 and 4 respectively [figure 1]. $20 \%$ (n-6) patients had previously taken PEG-IFN and Ribavirin treatment. All 30 patients achieved RVR and ETR (100\%). All of them also achieved SVR12 (100\%).

In the subgroup analysis, all (100\%) patients with genotype 1, 3 and genotype 4 achieved RVR, ETR and SVR12. The results were same for cirrhotic and non-cirrhotic patients as well as for naïve and treatment experienced patients (100\%) [Figure 2, 3, 4]

We reviewed major trials available till date for similar therapy. Type of treatment, study population, RVR and SVR12 rates of these trials are shown in table 2 .

Cohorts which were given SPR regimen in these trials had RVR rate $>94 \%$ with SVR12 rates ranging from $89-100 \%$ [Table 1]. Interesting thing to note in Electron study is, 2 groups which were given substandard treatment as per present recommendations $[1,2]$ like Sofosbuvir monotherapy in genotype 3 or Sofosbuvir and Ribavirin for treatment experienced genotype 1 patients, also achieved $100 \%$ RVR rates, although SVR12 rates were $60 \%$ and 10\% respectively [9]. In Boson Study, SVR12 in SPR group was $93 \%$ with high on-treatment responses [10].
Table 1: Baseline characteristics of the patients in patients on therapy containing Sofosbuvir, PEG-IFN and Ribavirin (SPR) (n- number; SDstandard deviation; HCV RNA- hepatitis $\mathrm{C}$ virus ribonucleic acid; IU/mlinternational units per millilitre).

\section{BASELINE PATIENT CHARACTERISTICS IN THE SPR REGIMEN}

\begin{tabular}{|l|l|}
\hline Number of patients (n) & 30 \\
\hline Age (years) Mean \pm SD & $47.7 \pm 10.8$ \\
\hline Sex (n/\%) & \\
\hline Male & $6(20 \%)$ \\
\hline Female & $24(80 \%)$ \\
\hline Genotype (n/\%) & \\
\hline 1 & $12(40 \%)$ \\
\hline 3 & $17(57 \%)$ \\
\hline 4 & $1(3 \%)$ \\
\hline HCV RNA baseline (IU/ml) Mean & $24,07,530$ \\
\hline Patients with cirrhosis (n/\%) & $17(57 \%)$ \\
\hline Treatment history (n/\%) & \\
\hline Naïve & $24(80 \%)$ \\
\hline Experienced & $6(20 \%)$ \\
\hline RVR achieved (n/\%) & $30(100 \%)$ \\
\hline ETR achieved (n/\%) & $30(100 \%)$ \\
\hline SVR 12 (n/\%) & $30(100 \%)$ \\
\hline
\end{tabular}

Genotype distribution in SPR Regimen

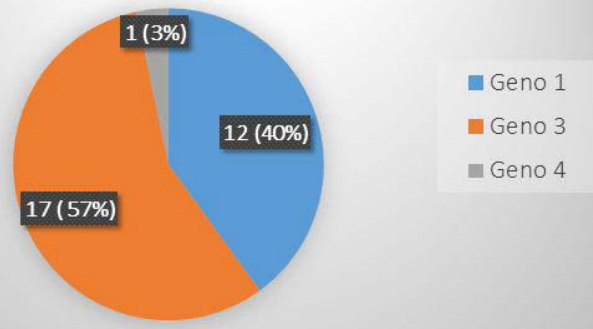

Figure 1: Distribution of patients according to genotype in SPR regimen cohort in our data. (SPR- sofosbuvir + PEG-IFN + Ribavirin). Majority patients were having genotype $3(57 \%)$ as commonly seen in India.

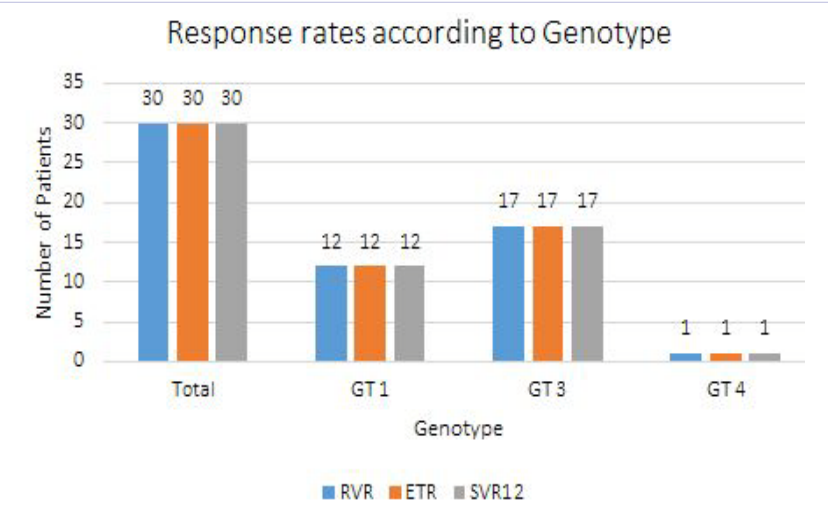

Figure 2: Number of patients achieving RVR, ETR and SVR12 according to genotypes. (RVR- rapid virological response; ETR- end of treatment response; SVR12- sustained virological response at week 12; GT- genotype) 


\section{Discussion}

Retrospective data from our centre showed 100\% RVR at week 4, ETR and SVR12 in patients treated with Sofosbuvir, PEGIFN and Ribavirin for 12 weeks, irrespective of their genotype, presence of cirrhosis or history of previous treatment failure. There was no availability of control subgroups. Due to $100 \%$ RVR and SVR12 rates, predictive relation between them could not be derived.

In Comparison with other available trials, Lawitz E et al in Neutrino study [6] concluded that since virologic suppression was achieved by week 4 in almost all patients and was maintained until the end of treatment, response guided treatment was not required in Sofosbuvir. Another trial by Lawitz E et al [7] did not find any clear predictors of treatment failure due to very small number of relapsers. Only 2 patients failed to achieve RVR but both of them achieved ETR and SVR. Atomic trial [8] has shown uniformly high rates of RVR and SVR12 with SPR regimen.

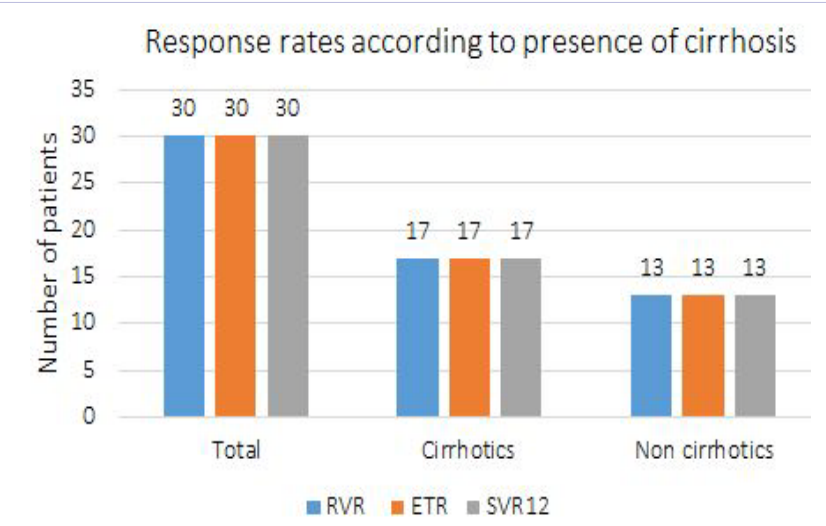

Figure 3: Number of patients achieving RVR, ETR and SVR12 according to presence or absence of cirrhosis. (RVR- rapid virological response; ETR- end of treatment response; SVR12- sustained virological response at week 12; GT- genotype)

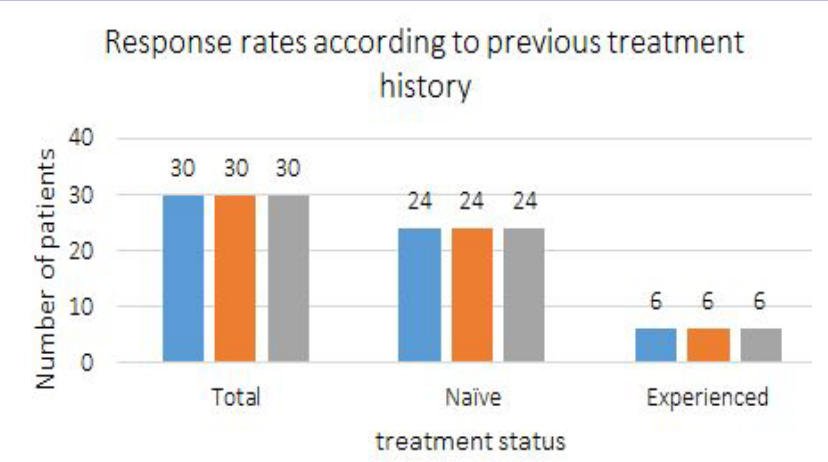

mVVR $=\mathrm{ETR}=\mathrm{SVR} 12$

Figure 4: Number of patients achieving RVR, ETR and SVR12 according to history of previous treatment. (RVR- rapid virological response; ETR- end of treatment response; SVR12- sustained virological response at week 12; GT- genotype)
Table 2: Type of treatment given, study population, RVR and SVR12 rates in trials of therapy containing Sofosbuvir, PEG-IFN and Ribavirin (SPR) for 12 weeks. (TN- treatment naïve, TE- treatment experienced, w- weeks), *HCV RNA at 8 weeks of treatment as 4 weeks data is not provided.

\begin{tabular}{|l|l|l|l|l|l|}
\hline Study & $\begin{array}{l}\text { Treatment } \\
\text { regimen }\end{array}$ & $\begin{array}{l}\text { Number of } \\
\text { patients (n) }\end{array}$ & Genotype & $\begin{array}{l}\text { RVR } \\
\text { (\%) }\end{array}$ & $\begin{array}{l}\text { SVR12 } \\
\text { (\%) }\end{array}$ \\
\hline Neutrino [6] & SPR-12 w & 327 & $1,4 \mathrm{TN}$ & 99 & 90 \\
\hline $\begin{array}{l}\text { Lonestar 2 } \\
{[7]}\end{array}$ & SPR-12 w & 47 & $2,3 \mathrm{TE}$ & 96 & 89 \\
\hline Atomic [8] & SPR-12 w & 52 & $1 \mathrm{TN}$ & 94 & 90 \\
\hline & SPR- 24 w & 125 & $1,4,6 \mathrm{TN}$ & 98 & 93 \\
\hline Electron [9] & SPR-12 w & 11 & 2,3 & 100 & 100 \\
\hline & SPR- 8 w & 10 & 2,3 & 100 & 100 \\
\hline Boson [10] & SPR-12 w & 94 & $3, \mathrm{TN}$ & $99 *$ & 95 \\
\hline & SPR-12 w & 87 & $3, \mathrm{TE}$ & $99 *$ & 91 \\
\hline
\end{tabular}

Authors concluded that there would be no need to tailor either treatment duration or regimen on the bases of early response like RVR and response guided treatment is not needed for treatment with Sofosbuvir. In Boson study, among those who relapsed, $38 \%$ of those who received 16 weeks and $33 \%$ of those who received 24 weeks of SR had HCV RNA undetectable at week 2 of treatment, as compared with 61\% (P - 0.007) and 58\% (P - 0.028) of those who achieved SVR12. At week 4, this difference was not significant. Among patients receiving SPR regimen, on treatment response among those who relapsed and those who achieved SVR12 was similar [10].

Till date, no data have emerged to suggest that those with detectable HCV RNA at 4 weeks will not attain SVR. Multiple studies have been carried out for treatment of sofosbuvir with rates of SVR1 2 ranging from $10 \%$ to $100 \%$, even though RVR rates were $>95 \%$ [6-11]. This clearly suggests that achievement of RVR has no relation with the achievement of SVR12. Achievement of RVR does not make any difference in treatment duration or regimen. According to recommendations by major associations, RVR is useful only to check compliance of the patient in the era of newer DAAs [1,2], but compliance can be assessed and increased by improving patient education and proper patient counselling instead of spending for the expensive test like HCV RNA. Limitations of our study were small sample size. Although RVR rates would be similar even with large sample size as seen in the above mentioned trials; less likely to affect the end results. So we conclude that pre-treatment HCV RNA and demonstrating its absence at 12 weeks after the end of therapy may suffice in treatment with Sofosbuvir especially in India.

\section{References}

1. AASLD/IDSA HCV Guidance Panel. Hepatitis C guidance: AASLD-IDSA recommendations for testing, managing, and treating adults infected with hepatitis C virus. Hepatology. 2015;62(3):932-954.

2. European Association for Study of Liver. EASL Recommendations on Treatment of Hepatitis C 2015. J Hepatol. 2015;63(1):199-236.

3. Fried MW, Hadziyannis SJ, Shiffman ML, Messinger D, Zeuzem S. Rapid virological response is the most important predictor of 
sustained virological response across genotypes in patients with chronic hepatitis C virus infection. J Hepatol. 2011;55(1):69-75. Doi: 10.1016/j.jhep.2010.10.032.

4. Mangia A, Santoro R, Minerva N, Ricci GL, Carretta V, Persico M, et al. Peg-interferon alfa-2b and ribavirin for 12 vs. 24 weeks in HCV genotype 2 or 3. N Engl J Med. 2005;352(25):2609-2617.

5. Dhiman RK. Future of therapy for Hepatitis $C$ in India: A Matter of Accessibility and Affordability? J Clin Exp Hepatol. 2014;4(2):85-86. Doi: 10.1016/j.jceh.2014.06.011.

6. Lawitz E, Mangia A, Wyles D, Maribel Rodriguez-Torres, Tarek Hassanein, Stuart C. Gordon, et al. Sofosbuvir for previously untreated chronic hepatitis C infection. N Engl J Med. 2013;368(20):1878-87.

7. Lawitz E, Poordad F, Brainard DM, Symonds WT, McHutchison JG, Membreno FE, et al. Sofosbuvir with peginterferon-ribavirin for 12 weeks in previously treated patients with hepatitis C genotype 2 or 3 and cirrhosis. Hepatology. 2015;61(3):769-75. Doi: 10.1002/ hep.27567.
8. Kowdley KV, Lawitz E, Crespo I, Hassanein T, Davis MN, DeMicco $M$, et al. Sofosbuvir with pegylated interferon alfa-2a and ribavirin for treatment-naïve patients with hepatitis $C$ genotype- 1 infection (ATOMIC): an open-label, randomised, multicentre phase 2 trial. Lancet. 2013;381(9883):2100-2107.

9. Gane EJ, Stedman CA, Hyland RH, Xiao Ding, Evguenia Svarovskaia, William T. Symonds, et al. Nucleotide polymerase inhibitor sofosbuvir plus ribavirin for hepatitis C. N Engl J Med. 2013;368(1):34-44.

10. Foster GR, Pianko S, Forton D, Nahass RG, George J, Brown A, et al. BOSON Study Group. Efficacy of Sofosbuvir plus Ribavirin With or Without Peginterferon-Alfa in Patients with Hepatitis C Virus Genotype 3 Infection and Treatment-Experienced Patients with Cirrhosis and Hepatitis C Virus Genotype 2 Infection. Gastroenterology. 2015;149(6):1462-1470. Doi: 10.1053/j.gastro.2015.07.043. 